\title{
Making Holes in the Second Symmetric Product of a Cyclicly Connected Graph
}

\author{
José G. Anaya ${ }^{1}$, David Maya ${ }^{1} \&$ Fernando Orozco-Zitli $^{1}$ \\ ${ }^{1}$ Universidad Autónoma del Estado de México, Facultad de Ciencias, Toluca, México \\ Correspondence: David Maya, Universidad Autónoma del Estado de México, Facultad de Ciencias, Instituto Lit- \\ erario 100. Col. Centro, C. P. 50000, Toluca, Estado de México, México. E-mail: dmayae@uaemex.mx
}

Received: June 9, 2014 Accepted: July 2, 2014 Online Published: August 4, 2014

doi:10.5539/jmr.v6n3p105

URL: http://dx.doi.org/10.5539/jmr.v6n3p105

\begin{abstract}
A continuum is a connected compact metric space. The second symmetric product of a continuum $X, \mathcal{F}_{2}(X)$, is the hyperspace of all nonempty subsets of $X$ having at most two elements. An element $A$ of $\mathcal{F}_{2}(X)$ is said to make a hole with respect to multicoherence degree in $\mathcal{F}_{2}(X)$ if the multicoherence degree of $\mathcal{F}_{2}(X)-\{A\}$ is greater than the multicoherence degree of $\mathcal{F}_{2}(X)$. In this paper, we characterize those elements $A \in \mathcal{F}_{2}(X)$ such that $A$ makes a hole with respect to multicoherence degree in $\mathcal{F}_{2}(X)$ when $X$ is a cyclicly connected graph.
\end{abstract}

Keywords: continuum, symmetric products, multicoherence degree, make a hole with respect to multicoherence degree

\section{Introduction}

A continuum is a connected compact metric space. Let $X$ be a continuum. For each positive interger $n$, let $\mathcal{F}_{n}(X)=\{A \subset X: A$ has at most $n$ elements and $A \neq \emptyset\}$. The hyperspace $\mathcal{F}_{n}(X)$ is called the $n^{\text {th }}$ symmetric product of $X$. It is known that each hyperspace $\mathcal{F}_{n}(X)$ is a continuum (see Borsuk \& Ulam, 1931, pp. 876, 877) and (Michael, 1951, Theorem 4.10, p. 165).

If $Z$ is any topological space, let $b_{0}(Z)$ denote the number of components of $Z$ minus one if this number is finite and $b_{0}(Z)=\infty$ otherwise. Given a connected topological space $Y$, the multicoherence degree of $Y$, is defined by $r(Y)=\sup \left\{b_{0}(K \cap L): K\right.$ and $L$ are closed connected subsets of $Y$ and $\left.Y=K \cup L\right\}$. The space $Y$ is said to be unicoherent if $r(Y)=0$. Let $y \in Y$ such that $Y-\{y\}$ is connected, we say that $y$ makes a hole with respect to multicoherence degree in $Y$ if $r(Y-\{y\})>r(Y)$. This is a generalization of the notion of to make a hole in a unicoherent topological space defined in (Anaya, 2007, p. 2000).

In this paper, we are interesting in the following problem.

Problem. Let $\mathcal{H}(X)$ be a hyperspace of a continuum $X$. For which elements $A \in \mathcal{H}(X), A$ makes a hole with respect to multicoherence degree in $\mathcal{H}(X)$.

In the current paper, we are presenting the solution to this problem when $X$ is a cyclicly connected graph and $\mathcal{H}(X)=\mathcal{F}_{2}(X)$.

Readers specially interested in this problem are refered to Anaya (2007, 2011), Anaya, Maya and Orozco-Zitli (2010, 2012).

\section{Preliminaries}

Given a positive interger $m$, define $\lambda(m)=\{1,2, \ldots, m\}$. A map is a continuous function. The identity map for a topological space $Z$ is denoted by $\mathrm{id}_{Z}$. An arc is any space homeomorphic to [0,1]. A simple closed curve is a space which is homeomorphic to the unit circle $S^{1}$ in the Euclidean plane $\mathbb{R}^{2}$. A theta curve is a space which is homeomorphic to $S^{1} \cup([-1,1] \times\{0\})$ in $\mathbb{R}^{2}$. The symbol $[0,1]^{2}$ denotes the space $[0,1] \times[0,1]$. The set $\left\{(u, v) \in[0,1]^{2}: u \leq v\right\}$ is denoted by $\Delta$. A graph is a continuum which can be written as the union of finitely many arcs any two of which are either disjoint or intersect only in one or both their end points. A point $y$ in a connected topological space $Y$ is called cut point (non-cut point) if $Y-\{y\}$ is not connected (connected). A space $W$ is said to be cyclicly connected provided that every two points of $W$ belong to some simple closed curve in $W$ 
(see (Whyburn, 1942, p. 77)). A graph $X$ is a cyclicly connected graph if $X$ is a cyclicly connected space.

Given a topological space $Y$. A subspace $Z$ of $Y$ is said to be:

(a) a retract of $Y$ if there exists a map $f: Y \rightarrow Z$ such that $f(z)=z$ for every $z \in Z$. The map $f$ is called a retraction.

(b) a deformation retract of $Y$ if there exist a retraction $f: Y \rightarrow Z$ and a map $g: Y \times[0,1] \rightarrow Y$ such that $g(x, 0)=x$ and $g(x, 1)=f(x)$ for every $x \in Y$.

(c) a strong deformation retract of $Y$ if there exist $f$ and $g$ as in (b) with the additional property that $g(z, t)=z$ for every $(z, t) \in Z \times[0,1]$.

Let $y \in Y$. Let $\beta$ be a cardinal number. We say that $y$ is of order less than or equal to $\beta$ in $Y$, written $\operatorname{ord}(y, Y) \leq \beta$, provided that for each open subset $U$ of $Y$ containing $y$, there exists an open subset $V$ of $Y$ such that $y \in V \subset U$ and the cardinality of the boundary of $V$ is less than or equal to $\beta$. We say that $y$ is of order $\beta$ in $Y$, written $\operatorname{ord}(y, Y)=\beta$, provided that $\operatorname{ord}(y, Y) \leq \beta$ and $\operatorname{ord}(y, Y) \not \leq \alpha$ for any cardinal number $\alpha<\beta$. Put $E(Y)=\{x \in Y$ : $\operatorname{ord}(x, Y)=1\}, O(Y)=\{x \in Y: \operatorname{ord}(x, Y)=2\}$ and $R(Y)=\{x \in Y: \operatorname{ord}(x, Y) \geq 3\}$. Define $\mathcal{I}(Y)=\{I \subset Y:$ $I$ is an arc and $E(I)=I \cap R(Y)\}, \mathcal{N}(y, Y)=\{I \in \mathcal{I}(Y): y \notin I\}, \mathcal{M}(y, Y)=\{I \in \mathcal{I}(Y): y \in I\}, N(y, Y)=\bigcup \mathcal{N}(y, Y)$ and $M(y, Y)=\bigcup \mathcal{M}(y, Y)$. If $K$ and $L$ are nonempty subsets of $Y$, let $\langle K, L\rangle=\{\{x, y\} \subset Y: x \in K, y \in L\}$.

\subsection{Auxiliary Results}

Lemma 2.1 If $X$ is a cyclicly connected graph different from a simple closed curve, then the following conditions hold:

(1) for each simple closed curve $S$ in $X, S \cap R(X)$ has at least two points;

(2) $X=\cup I(X)$;

(3) the set $\mathcal{I}(X)$ is finite;

(4) for each $p \in X, M(p, X)$ is a nondegenerate subcontinuum of $X$.

Proof. In order to prove (1), let $S$ be a simple closed curve in $X$. Since $S \neq X$, there exists a simple closed curve $S_{1} \neq S$ in $X$ such that $S \cap S_{1} \neq \emptyset$. So, using (Nadler, Jr., 1992, Proposition 9.5, p. 142), $R\left(S \cup S_{1}\right) \cap S \cap S_{1} \neq \emptyset$. Thus, by (Kuratowski, 1968, Theorem 3, p. 278), $R(X) \cap S \cap S_{1} \neq \emptyset$. Now, assume that $R(X) \cap S \cap S_{1}$ consists of precisely one point. Then, there exists a simple closed curve $S_{2} \neq S$ in $X$ such that $S_{2} \cap\left(S-S_{1}\right) \neq \emptyset$. Applying the previous argument to $S \cup S_{2}$, we have $R(X) \cap\left(S-S_{1}\right) \cap S_{2} \neq \emptyset$. Hence, $S \cap R(X)$ has at least two points.

(2) Follows from (1) and the fact that $R(X)$ is a finite set (see (Nadler, Jr., 1992, Theorem 9.10, p. 144)).

(3) Follows from the fact that $R(X)$ is a finite set (see (Nadler, Jr., 1992, Theorem 9.10, p. 144)).

Finally, to check (4), let $p \in X$. By (2), there exists $I \in \mathcal{I}(X)$ such that $p \in I$. So, since $I \subset M(p, X), M(p, X)$ is nondegenerate set. On the other hand, clearly, $M(p, X)$ is connected. By (3), $M(p, X)$ is closed in $X$.

Lemma 2.2 Let $X$ be a cyclicly connected graph and let $p \in X$. If $\mathcal{N}(p, X) \neq \emptyset$, then $N(p, X)$ is a subcontinuum of $X$.

Proof. First, by (3) of Lemma 2.1, $N(p, X)$ is closed in $X$. We shall prove the connectedness of $N(p, X)$. By (Whyburn, 1942, (9.3), p. 79), $X-\{p\}$ is connected. So, it suffices to prove that $N(p, X)$ is a continuous image of $X-\{p\}$. Consider $F=\bigcup\{E(I): I \in \mathcal{M}(p, X)\}-\{p\}$. By (3) of Lemma 2.1, $\mathcal{M}(p, X)$ is finite. Then, $F$ is discrete. By (4) of Lemma 2.1, $M(p, X)-\{p\}$ is a nonempty set. Now, define $f: M(p, X)-\{p\} \rightarrow F$ as follows: given $z \in M(p, X)-\{p\}$, let $f(z)$ be the unique element of $F \cap C$ where $C$ is the component of $M(p, X)-\{p\}$ containing $z$. Clearly, $f$ is surjective. We prove that $f$ is continuous. Let $e \in F$. By the definition of $f$, it is easy to see that $f^{-1}(\{e\})$ is a component of $M(p, X)-\{p\}$. Thus, since each component of $M(p, X)-\{p\}$ is closed in $M(p, X)-\{p\}$, $f^{-1}(\{e\})$ is closed in $M(p, X)-\{p\}$.

Now, define $\bar{f}: X-\{p\} \rightarrow N(p, X)$ by

$$
\bar{f}(x)=\left\{\begin{array}{cl}
x, & \text { if } x \in N(p, X), \\
f(x), & \text { if } x \in M(p, X)-\{p\} .
\end{array}\right.
$$

Since $N(p, X) \cap M(p, X)=F$ and by the definition of $f, \bar{f}$ is well defined. Clearly, $\bar{f}$ is surjective. The continuity of $\bar{f}$ follows from the continuity of $f$ and the fact that $N(p, X)$ and $M(p, X)-\{p\}$ are closed subsets of $X-\{p\}$. This finishes the proof of that $N(p, X)$ is connected. 
Lemma 2.3 Let $X$ be a cyclicly connected graph different from a simple closed curve and let $p, q$ be different points in $X$. If $X-\{p, q\}$ is not connected, there exist a simple closed curve $S$ in $X$ containing $p$ and $q$ and a retract $f$ : $X \rightarrow S$ such that $f^{-1}(p)=\{p\}$ and $f^{-1}(q)=\{q\}$.

Proof. Let $C_{1}$ and $C_{2}$ be different components of $X-\{p, q\}$. Since $C_{k} \cup\{p, q\}$ is a subcontinuum of $X$, there exists an arc $J_{k}$ in $C_{k} \cup\{p, q\}$ such that $E\left(J_{k}\right)=\{p, q\}$ for each $k \in\{1,2\}$. Put $S=J_{1} \cup J_{2}$. Clearly, $S$ is a simple closed curve in $X$ and $p, q \in S$.

Now, let $f_{0}: R(X) \rightarrow S$ be a function such that $\left.f_{0}\right|_{R(X) \cap S}=\operatorname{id}_{R(X) \cap S}, f_{0}\left(R(X) \cap C_{1}\right) \subset J_{1}$ and $f_{0}(R(X) \cap C) \subset J_{2}$ for each component $C$ of $X-\{p, q\}$ with $C \neq C_{1}$.

Given $I \in \mathcal{I}(X)$, let $f_{I}: I \rightarrow S$ be a one-to-one map such that $\left.f_{I}\right|_{S}=\mathrm{id}_{S \cap I}, E\left(f_{I}(I)\right)=f_{0}(E(I)), f_{I}\left(I \cap C_{1}\right) \subset J_{1}$ and $f_{I}(I \cap C) \subset J_{2}$ for each component $C$ of $X-\{p, q\}$ with $C \neq C_{1}$. From the fact that $f_{I}$ is one-to-one, it follows that $f_{I}(I-\{p, q\}) \subset S-\{p, q\}$.

Define $f: X \rightarrow S$ as follows: for each $x \in X$, take $I \in I(X)$ such that $x \in I$ and let $f(x)=f_{I}(x)$. Notice that $\left.f\right|_{R(X)}=f_{0}$. Hence, $f$ is well defined. The continuity of $f$ follows from the fact that each $f_{I}$ is continuous and, by (2) and (3) of Lemma 2.1. It is easy to see that $\left.f\right|_{S}=\mathrm{id}_{S}$. Thus, $f$ is a retraction.

Finally, since $S-\{p, q\} \subset X-\{p, q\}$ and $\left.f\right|_{S}=\operatorname{id}_{S}, S-\{p, q\} \subset f(X-\{p, q\})$. To check that $f(X-\{p, q\}) \subset$ $S-\{p, q\}$, notice that $f(X-\{p, q\})=\bigcup\left\{f_{I}(I-\{p, q\}): I \in I(X)\right\} \subset S-\{p, q\}$ (see (2). of Lemma 2.1). Thus, $f(X-\{p, q\})=S-\{p, q\}$. Hence, $f^{-1}(\{p, q\})=\{p, q\}$. From the fact that $p \neq q$, we have that $f^{-1}(p)=\{p\}$ and $f^{-1}(q)=\{q\}$

Lemma 2.4 Let $X$ be a cyclicly connected graph different from a simple closed curve and let $p, q$ be different points in $X$. If $X-\{p, q\}$ is connected, there exist a theta curve $Y$ in $X$ containing $p$ and $q$ and a retract $f: X \rightarrow Y$ such that $f^{-1}(p)=\{p\}$ and $f^{-1}(q)=\{q\}$.

Proof. By the definition of cyclic connectedness, there exists a simple closed curve $S$ in $X$ such that $p, q \in Y$. Since $X-\{p, q\}$ is connected, there exists an arc $J$ in $X$ such that $S-\{p, q\} \cap J=E(J)$. Put $Y=S \cup J$. Clearly, $Y$ is a theta curve in $X$ containing $p$ and $q$ such that $Y-\{p, q\}$ is connected.

First, consider a function $f_{0}: R(X) \rightarrow Y$ such that $\left.f_{0}\right|_{Y}=\operatorname{id}_{R(X) \cap Y}$. Now, for each $I \in \mathcal{I}(X)$, fix a one-to-one map $f_{I}$ : $I \rightarrow Y$ such that $\left.f_{I}\right|_{Y}=\operatorname{id}_{Y \cap I}$ and $f(I-\{p, q\}) \subset Y-\{p, q\}$.

Define $f: X \rightarrow Y$ as follows: for each $x \in X$, take $I \in \mathcal{I}(X)$ such that $x \in I$ and let $f(x)=f_{I}(x)$. From the fact that $\left.f\right|_{R(X)}=f_{0}$, it follows that $f$ is well defined. Since $X=\bigcup \mathcal{I}(X)$ and $\mathcal{I}(X)$ is finite (see (2) and (3) of Lemma 2.1), $f$ is continuous. From the fact that $\left.f\right|_{Y}=\operatorname{id}_{Y}$, it follows that $f$ is a retraction.

We will prove that $f(X-\{p, q\})=Y-\{p, q\}$. Since $X-\{p, q\}=\bigcup\{I-\{p, q\}: I \in I(X)\}, f(X-\{p, q\}) \subset Y-\{p, q\}$. Clearly, $Y-\{p, q\}$ is contained in $f(X-\{p, q\})$. We have that $f^{-1}(\{p, q\})=\{p, q\}$. Since $p \neq q, f^{-1}(p)=\{p\}$ and $f^{-1}(q)=\{q\}$

Proposition 2.5 Let $X$ be a continuum and let $K$ and $L$ be connected subsets (subcontinua) of $X$. Then $\langle K, L\rangle$ is a connected subset (subcontinuum) of $\mathcal{F}_{2}(X)$ and, it does not have cut points when $K$ and L are nondegenerate sets.

Proof. The connectedness of $\langle K, L\rangle$ follows from (Martínez-Montejano, 2002, Lemma 1, p. 230).

In order to prove the second part of this proposition, let $\{p, q\} \in\langle K, L\rangle$. Using $K$ and $L$ are nondegenerate sets and the arguments in (Kuratowski, 1968, Theorem 11, p. 137), it can be shown that $K \times L-\{(p, q),(q, p)\}$ is connected. So, since $\langle K, L\rangle-\{\{p, q\}\}$ is a continuous image of $K \times L-\{(p, q),(q, p)\},\langle K, L\rangle-\{\{p, q\}\}$ is connected.

Lemma 2.6 Let $I$ be an arc and let $p \in I-E(I)$. If $H$ and $J$ are subcontinua of I such that $H \cup J \subset I-\{p\}$ and each one of them contains a different end point of $I$, then $\langle H, I\rangle \cup\langle J, I\rangle$ is a strong deformation retract of $\mathcal{F}_{2}(I)-\{\{p\}\}$.

Proof. Put $\Gamma=\Delta-\left\{\left(\frac{1}{2}, \frac{1}{2}\right)\right\}, \Gamma_{0}=\left\{(u, v) \in \Gamma: u \leq \frac{1}{4}\right\} \cup\left\{(u, v) \in \Gamma: \frac{3}{4} \leq v\right\}$ and $\Gamma_{1}=\left\{(u, v) \in \Gamma: \frac{1}{4} \leq u, v \leq \frac{3}{4}\right\}$. First, we are going to prove that $\Gamma_{0}$ is a strong deformation retract of $\Gamma$. Define $f: \Gamma \rightarrow \Gamma_{0}$ by

$$
f(u, v)=\left\{\begin{array}{cl}
(u, v), & \text { if }(u, v) \in \Gamma_{0}, \\
\left(\frac{1}{4}, u+v-\frac{1}{4}\right), & \text { if }(u, v) \in \Gamma_{1} \text { and } v \leq 1-u \\
\left(u+v-\frac{3}{4}, \frac{3}{4}\right), & \text { if }(u, v) \in \Gamma_{1} \text { and } 1-u \leq v,
\end{array}\right.
$$

and $g: \Gamma \times[0,1] \rightarrow \Gamma$ by

$$
g((u, v), t)=(1-t) \cdot(u, v)+t \cdot f(u, v) .
$$


It is easy to verify that $f$ and $g$ have the required properties.

Finally, let $h:[0,1] \rightarrow I$ be a homeomorphism such that $h\left(\left[0, \frac{1}{4}\right]\right)=H, h\left(\left[\frac{3}{4}, 1\right]\right)=J$ and $h\left(\frac{1}{2}\right)=p$. Define $\bar{h}: \Gamma \rightarrow$ $\mathcal{F}_{2}(I)-\{\{p\}\}$ by $\bar{h}(u, v)=\{h(u), h(v)\}$. It can be proved that $\bar{h}$ is a homeomorphism such that $\bar{h}\left(\Gamma_{0}\right)=\langle H, I\rangle \cup\langle J, I\rangle$. Therefore, $\langle H, I\rangle \cup\langle J, I\rangle$ is a strong deformation retract of $\mathcal{F}_{2}(I)-\{\{p\}\}$.

Lemma 2.7 If $X$ is a graph containing a simple closed curve, then $X$ is not unicoherent.

Proof. We shall prove that there exist subcontinua $K$ and $L$ of $X$ such that $b_{0}(K \cap L)>0$ and $X=K \cup L$. Let $S$ be a simple closed curve in $X$. By (Nadler, Jr., 1992, Theorem 9.10, p. 144), there exists $x \in S$ such that ord $(x, X)=2$. Now, using (Nadler, Jr., 1992, Theorem 9.7, p. 143), it can be proved that there exists an arc $J$ in $S$ which is a neighborhood of $x$ in $X$. Then, $J-E(J)$ is an open connected subset of $X$. Now, by (Nadler, Jr., 1992, 9.44, (a), p. 160), $S-(J-E(J))$ is connected. Hence, $X-(J-E(J))$ is a subcontinuum of $X$. So, $K=J$ and $L=X-(J-E(J))$ satify the requiered properties.

Theorem 2.8 If $X$ is a cyclicly connected graph, then $r\left(\mathcal{F}_{2}(X)\right)=1$.

Proof. The result follows from (Nadler, Jr., 1992, Theorem 8.25, p. 131), Lemma 2.7 and (Illanes, 1985, Theorem 1.6, p. 16).

\section{Making Holes in the Second Symmetric Product of a Cyclicly Connected Graph}

Theorem 3.1 Let $X$ be a graph and let $p \in O(X)$. Then $\{p\}$ does not make a hole with respect to multicoherence degree in $\mathcal{F}_{2}(X)$.

Proof. We will show that $r\left(\mathcal{F}_{2}(X)-\{\{p\}\}\right)=r\left(\mathcal{F}_{2}(X)\right)$. Since $X$ is a graph, it is easy to see that $\mathcal{F}_{2}(X)-\{\{p\}\}$ is a locally connected metric space and, by Proposition 2.5, $\mathcal{F}_{2}(X)-\{\{p\}\}$ is connected. So, in light of (Eilenberg, 1936, Theorem 4, p. 162) and (Stone, 1950, Theorem 5, p. 472), it suffices to prove that there exists a deformation retract $\mathcal{Z}$ of $\mathcal{F}_{2}(X)-\{\{p\}\}$ such that $r(\mathcal{Z})=r\left(\mathcal{F}_{2}(X)\right)$.

Since $p \in O(X)$, using (Nadler, Jr., 1992, Lemma 9.7, p. 143), it can be shown that there exists an arc $I$ in $X$ such that $I$ is a neighborhood of $p$ in $X$. So, clearly, $p \in I-E(I)$. Let $H$ and $J$ be nondegenerate subcontinua of $I$ such that $H \cup J \subset I-\{p\}$ and each one of them contains a different end point of $I$. Put $Z=(X-I) \cup H \cup J$ and $\mathcal{Z}=\langle X, Z\rangle$. Clearly, $\mathcal{F}_{2}(X)=\mathcal{Z} \cup \mathcal{F}_{2}(I)$. Now, by Lemma 2.6, there exist a retraction $f: \mathcal{F}_{2}(I)-\{\{p\}\} \rightarrow\langle H, I\rangle \cup\langle J, I\rangle$ and a $\operatorname{map} g:\left(\mathcal{F}_{2}(I)-\{\{p\}\}\right) \times[0,1] \rightarrow \mathcal{F}_{2}(I)-\{\{p\}\}$ such that $g(A, 0)=A$ and $g(A, 1)=f(A)$ for each $A \in \mathcal{F}_{2}(I)-\{\{p\}\}$ and $g(B, t)=B$ for each $(B, t) \in(\langle H, I\rangle \cup\langle J, I\rangle) \times[0,1]$.

Define $\bar{f}: \mathcal{F}_{2}(X)-\{\{p\}\} \rightarrow \mathcal{Z}$ by

$$
\bar{f}(A)=\left\{\begin{array}{cl}
A, & \text { if } A \in \mathcal{Z}, \\
f(A), & \text { if } A \in \mathcal{F}_{2}(I)-\{\{p\}\},
\end{array}\right.
$$

and $\bar{g}:\left(\mathcal{F}_{2}(X)-\{\{p\}\}\right) \times[0,1] \rightarrow \mathcal{F}_{2}(X)-\{\{p\}\}$ by

$$
\bar{g}(A, t)=\left\{\begin{array}{cl}
A, & \text { if } A \in \mathcal{Z}, \\
g(A, t), & \text { if } A \in \mathcal{F}_{2}(I)-\{\{p\}\} .
\end{array}\right.
$$

To check that $\bar{f}$ and $\bar{g}$ are well defined, notice that $\mathcal{Z} \cap \mathcal{F}_{2}(I)-\{\{p\}\}=\langle H, I\rangle \cup\langle J, I\rangle$ and $f(B)=B=g(B, t)$ for each $(B, t) \in(\langle H, I\rangle \cup\langle J, I\rangle) \times[0,1]$. Now, the continuity of $\bar{f}$ and $\bar{g}$ follows from the continuity of the maps $f$ and $g$ and the fact that $\mathcal{Z}$ and $\mathcal{F}_{2}(I)-\{\{p\}\}$ are closed in $\mathcal{F}_{2}(X)-\{\{p\}\}$. It is easy to verify that $\bar{f}$ and $\bar{g}$ have the required properties. Thus, $\mathcal{Z}$ is a deformation retract of $\mathcal{F}_{2}(X)-\{\{p\}\}$.

Finally, to check that $r(\mathcal{Z})=r\left(\mathcal{F}_{2}(X)\right)$, we shall show that $\mathcal{Z}$ is homeomorphic to $\mathcal{F}_{2}(X)$. It can be shown that there exists a homeomorphism $h: \mathcal{F}_{2}(I) \rightarrow\langle H, I\rangle \cup\langle J, I\rangle$ such that $\left.h\right|_{\langle E(I), I\rangle}=\operatorname{id}_{\langle E(I), I\rangle}$. Define $\bar{h}: \mathcal{F}_{2}(X) \rightarrow \mathcal{Z}$ by

$$
\bar{h}(A)=\left\{\begin{array}{cl}
h(A), & \text { if } A \in \mathcal{F}_{2}(I), \\
A, & \text { otherwise. }
\end{array}\right.
$$

It is easy to see that $\bar{h}$ is a homeomorphism. Hence, $r\left(\mathcal{F}_{2}(X)\right)=r(\mathcal{Z})$.

This finishes the proof that $\{p\}$ does not make a hole with respect to multicoherence degree in $\mathcal{F}_{2}(X)$.

Theorem 3.2 Let $X$ be a cyclicly connected graph and $p \in R(X)$. Then $\{p\}$ makes a hole with respect to multicoherence degree in $\mathcal{F}_{2}(X)$. 
Proof. Since $r\left(\mathcal{F}_{2}(X)\right)=1$ (see Theorem 2.8), we shall show that $r\left(\mathcal{F}_{2}(X)-\{\{p\}\}\right) \geq 2$. So, it suffices to prove that there exist two closed connected subsets $\mathcal{K}$ and $\mathcal{L}$ of $\mathcal{F}_{2}(X)-\{\{p\}\}$ such that $\mathcal{F}_{2}(X)-\{\{p\}\}=\mathcal{K} \cup \mathcal{L}$ and $b_{0}(\mathcal{K} \cap \mathcal{L}) \geq 2$.

Put $\Lambda=\left\{(u, v) \in[0,1]^{2}-\{\mathbf{0}\}: \frac{u}{2} \leq v \leq 2 u\right\}, \Omega=\left\{(u, v) \in[0,1]^{2}-\{\mathbf{0}\}: v \leq \frac{u}{2}\right\}, \Gamma=\left\{(u, v) \in[0,1]^{2}-\{\mathbf{0}\}: 2 u \leq v\right\}$ where $\mathbf{0}=(0,0), m=\operatorname{ord}(p, X)$ and $\mathcal{M}(p, X)=\left\{I_{1}, I_{2}, \ldots, I_{m}\right\}$. For each $k \in \lambda(m)$, fix a homeomorphism $\varphi_{k}$ : $[0,1] \rightarrow I_{k}$ such that $\varphi_{k}(0)=p$. Given elements $k \neq j \in \lambda(m)$, define $\psi_{(k, j)}:[0,1]^{2}-\{\mathbf{0}\} \rightarrow\left\langle I_{k}, I_{j}\right\rangle-\{\{p\}\}$ by $\psi_{(k, j)}(s, t)=\left\{\varphi_{k}(s), \varphi_{j}(t)\right\}$. Since $\varphi_{k}(0)=\varphi_{j}(0)=p$ and, $\varphi_{k}$ and $\varphi_{j}$ are one-to-one, $\psi_{(k, j)}$ is well defined. Using the fact that $\varphi_{k}$ and $\varphi_{j}$ are surjective, it is easy to prove that $\psi_{(k, j)}$ is surjective. Clearly, for each $k, j \in \lambda(m)$ with $k \neq j$, $\psi_{(k, j)}(\Lambda)=\psi_{(j, k)}(\Lambda)$ and $\psi_{(k, j)}(\Omega)=\psi_{(j, k)}(\Gamma)$.

Consider the following cases.

Case A. $\mathcal{N}(p, X) \neq \emptyset$.

Let $Y=N(p, X)$. By Lemma 2.2, $Y$ is a subcontinuum of $X$. For each $k \in \lambda(m)$, define

$$
\mathcal{K}_{k}=\left\langle\varphi_{k}\left(\left[\frac{1}{2}, 1\right]\right), Y \cup \varphi_{k}\left(\left[\frac{1}{2}, 1\right]\right)\right\rangle \text { and } \mathcal{L}_{k}=\left\langle\varphi_{k}\left(\left[0, \frac{1}{2}\right]\right), Y \cup \varphi_{k}([0,1])\right\rangle-\{\{p\}\}
$$

Consider

$$
\begin{gathered}
\mathcal{K}=\mathcal{F}_{2}(Y) \cup \bigcup\left\{\mathcal{K}_{k}: k \in \lambda(m)\right\} \cup \bigcup\left\{\psi_{(k, j)}(\Lambda): k, j \in \lambda(m), k \neq j\right\} \\
\text { and } \mathcal{L}=\bigcup\left\{\mathcal{L}_{k}: k \in \lambda(m)\right\} \cup \bigcup\left\{\psi_{(k, j)}(\Gamma): k, j \in \lambda(m), k \neq j\right\}
\end{gathered}
$$

Clearly, $\mathcal{K}$ and $\mathcal{L}$ are closed subsets of $\mathcal{F}_{2}(X)-\{\{p\}\}$. To prove $\mathcal{F}_{2}(X)-\{\{p\}\}=\mathcal{K} \cup \mathcal{L}$, let $\{x, y\} \in \mathcal{F}_{2}(X)-\{\{p\}\}$. Since $X=M(p, X) \cup Y, \mathcal{F}_{2}(X)=\mathcal{F}_{2}(M(p, X)) \cup \mathcal{F}_{2}(Y) \cup\langle M(p, X), Y\rangle$. If $\{x, y\} \in \mathcal{F}_{2}(Y) \cup\langle M(p, X), Y\rangle$, it is easy to see that $\{x, y\} \in \mathcal{K} \cup \mathcal{L}$. Suppose that $\{x, y\} \in \mathcal{F}_{2}(M(p, X))-\{\{p\}\}$. Take $k, j \in \lambda(m)$ such that $x \in I_{k}$ and $y \in I_{j}$. First, if $k=j$, then $\{x, y\} \in \mathcal{K}_{k} \cup \mathcal{L}_{k}$. Now, without loss of generality, we may assume that $k<j$. Consider $(u, v) \in[0,1]^{2}-\{\mathbf{0}\}$ such that $\psi_{(k, j)}(u, v)=\{x, y\}$. Thus, since $[0,1]^{2}-\{\mathbf{0}\}=\Lambda \cup \Omega \cup \Gamma, \psi_{(k, j)}(\Lambda)=\psi_{(j, k)}(\Lambda)$ and $\psi_{(k, j)}(\Gamma)=\psi_{(j, k)}(\Omega),\{x, y\} \in \mathcal{K} \cup \mathcal{L}$.

To show that $\mathcal{K}$ and $\mathcal{L}$ are connected, let $k \neq j \in \lambda(m)$. The connectedness of $\mathcal{K}_{k}$ and $\mathcal{L}_{k}$ follows from the fact that $\varphi_{k}(1) \in Y$ and Proposition 2.5. Without loss of generality, we may assume that $k<j$. The connectedness of $\mathcal{L}$ follows from the connectedness of $\Omega$ and the fact that $\psi_{(k, j)}(1,0) \in \mathcal{L}_{k} \cap \mathcal{L}_{j} \cap \psi_{(k, j)}(\Omega)$. Since $\psi_{(k, j)}(\Lambda)$ is connected, $\psi_{(k, j)}(\Lambda)=\psi_{(j, k)}(\Lambda)$ and $\psi_{(k, j)}(1,1) \in \mathcal{K}_{k} \cap \psi_{(k, j)}(\Lambda) \cap \mathcal{F}_{2}(Y), \mathcal{K}$ is connected.

Finally, we will show that $b_{0}(\mathcal{K} \cap \mathcal{L}) \geq 2$. Put $\Sigma=\left\{(u, v) \in[0,1]^{2}-\{\mathbf{0}\}: v=2 u\right\}$. Given $k \in \lambda(m)$. Define $\mathcal{D}_{k}=\left\langle\left\{\varphi_{k}\left(\frac{1}{2}\right)\right\}, Y \cup \varphi_{k}\left(\left[\frac{1}{2}, 1\right]\right)\right\rangle$ and $C_{k}=\bigcup\left\{\psi_{(k, j)}(\Sigma): j \in \lambda(m)-\{k\}\right\} \cup \mathcal{D}_{k}$. We are going to prove that $C_{1}, \ldots, C_{m}$ are the components of $\mathcal{K} \cap \mathcal{L}$. The connectedness of $\mathcal{D}_{k}$ follows from the fact that $\varphi_{k}(1) \in Y$ and Proposition 2.5. Since $\psi_{(k, j)}(\Sigma)$ is connected and $\psi_{(k, j)}\left(\frac{1}{2}, 1\right) \in \psi_{(k, j)}(\Sigma) \cap \mathcal{D}_{k}$ for each $j \in \lambda(m)-\{k\}, C_{k}$ is connected.

We need to prove the following properties,

i) $\mathcal{F}_{2}(Y) \cap \mathcal{L}=\emptyset$,

ii) $\mathcal{K}_{k} \cap \mathcal{L}_{k}=\mathcal{D}_{k}$ for each $k \in \lambda(m)$,

iii) $\mathcal{K}_{k} \cap \mathcal{L}_{j}=\emptyset$ and $\mathcal{K}_{k} \cap \psi_{(k, j)}(\Gamma)=\left\{\varphi_{k}\left(\frac{1}{2}\right), \varphi_{j}(1)\right\}$ for each $k \neq j \in \lambda(m)$,

iv) $\mathcal{L} \cap\left\langle I_{k}, I_{j}\right\rangle=\psi_{(k, j)}(\Omega) \cup \psi_{(k, j)}(\Gamma)$ for each $k \neq j \in \lambda(m)$,

v) $\varphi_{k}([0,1]) \cap \varphi_{j}([0,1])=\left\{\varphi_{k}(0), \varphi_{k}(1)\right\} \cap\left\{\varphi_{j}(0), \varphi_{j}(1)\right\}$ for each $k \neq j \in \lambda(m)$,

vi) $\mathcal{D}_{k} \cap \mathcal{D}_{j}=\emptyset$ for each $k \neq j \in \lambda(m)$,

vii) if $k \neq j \in \lambda(m)$, then $\psi_{(k, j)}(\Sigma) \cap \mathcal{D}_{l}=\emptyset$ for each $l \in \lambda(m)-\{k\}$,

viii) if $k \neq j \in \lambda(m)$, then $\psi_{(k, j)}(\Sigma) \cap \psi_{(l, n)}(\Sigma)=\emptyset$ for each $(l, n) \in((\lambda(m)-\{k\}) \times(\lambda(m)-\{j\}))-\{(j, k)\}$.

It is easy to see the properties $i)-v)$.

vi) Follows from the facts that $\varphi_{k}\left(\frac{1}{2}\right) \notin Y \cup \varphi_{j}\left(\left[\frac{1}{2}, 1\right]\right), \varphi_{j}\left(\frac{1}{2}\right) \notin Y \cup \varphi_{k}\left(\left[\frac{1}{2}, 1\right]\right)$ and v).

vii) Suppose to the contrary that there exists $l \in \lambda(m)-\{k\}$ such that $\psi_{(k, j)}(\Sigma) \cap \mathcal{D}_{l} \neq \emptyset$. Consider $(u, 2 u) \in \Sigma$ such that $\psi_{(k, j)}(u, 2 u) \in \mathcal{D}_{l}$. Then, either $\varphi_{k}(u)=\varphi_{l}\left(\frac{1}{2}\right)$ or $\varphi_{j}(2 u)=\varphi_{l}\left(\frac{1}{2}\right)$. So, by v), $j=l$ and $\varphi_{j}(2 u)=\varphi_{l}\left(\frac{1}{2}\right)$. Thus, $u=\frac{1}{4}$ and $\varphi_{k}\left(\frac{1}{4}\right) \in Y \cup \varphi_{l}\left(\left[\frac{1}{2}, 1\right]\right)$, a contradiction. 
viii) Suppose to the contrary that there exist $(l, n) \in((\lambda(m)-\{k\}) \times(\lambda(m)-\{j\}))-\{(j, k)\}$ and $(u, v),(s, t) \in \Sigma$ such that $\psi_{(k, j)}(u, v)=\psi_{(l, n)}(s, t)$. So, since $u>0, s \leq \frac{1}{2}$ and $k \neq l$, by $\left.v\right), \varphi_{k}(u) \neq \varphi_{l}(s)$ and $\varphi_{k}(u)=\varphi_{n}(t)$. Then, $\varphi_{j}(v)=\varphi_{l}(s)$. Thus, since $0<u, s \leq \frac{1}{2}$, by v), $k=n$ and $j=l$. Hence, since $\varphi_{k}$ and $\varphi_{l}$ are one-to-one maps, $u=t$ and $v=s$. Therefore, $(t, s),(s, t) \in \Sigma$, a contradiction.

We are ready to prove that $\mathcal{K} \cap \mathcal{L}=\bigcup\left\{C_{k}: k \in \lambda(m)\right\}$. From the fact that $\Sigma=\Lambda \cap \Gamma$ and ii), we have that $\bigcup\left\{C_{k}\right.$ : $k \in \lambda(m)\} \subset \mathcal{K} \cap \mathcal{L}$. Now, let $\{w, z\} \in \mathcal{K} \cap \mathcal{L}$. If $\{w, z\} \in \mathcal{K}_{k} \cap \mathcal{L}$ for some $k \in \lambda(m)$, by ii) and iii), $\{w, z\} \in \mathcal{D}_{k} \subset C_{k}$. Now, suppose that $\{w, z\} \in \psi_{(k, j)}(\Lambda) \cap \mathcal{L}$ for some $k \neq j \in \lambda(m)$. Since $\psi_{(k, j)}(\Lambda) \subset\left\langle I_{k}, I_{j}\right\rangle$ and $\psi_{(k, j)}(\Gamma)=\psi_{(j, k)}(\Omega)$, by iv $),\{w, z\} \in\left(\psi_{(k, j)}(\Lambda) \cap \psi_{(k, j)}(\Omega)\right) \cup\left(\psi_{(k, j)}(\Lambda) \cap \psi_{(j, k)}(\Omega)\right)$. So, using $\Sigma=\Lambda \cap \Omega, \varphi_{(k, j)}(\Lambda)=\varphi_{(j, k)}(\Lambda)$ and $\left.\varphi_{(k, j)}\right|_{\Sigma},\left.\varphi_{(j, k)}\right|_{\Sigma}$ are one-to-one maps, it can be proved $\{w, z\} \in \varphi_{(k, j)}(\Sigma) \cup \varphi_{(j, k)}(\Sigma)$. Hence, $\{w, z\} \in C_{k} \cup C_{j}$.

Finally, in order to prove that $C_{1}, C_{2}, \ldots, C_{m}$ are mutually disjoint, let $k \neq j \in \lambda(m)$. By vi)-viii), $C_{k} \cap C_{j}=\emptyset$. Thus, $b_{0}(\mathcal{K} \cap \mathcal{L})+1=m \geq 3$.

Case B. $\mathcal{N}(p, X)=\emptyset$.

Then, $N(p, X)=\emptyset$ and $\varphi_{1}(1)=\varphi_{2}(1)=\cdots=\varphi_{k}(1)$. This case can be proved using similar arguments in the proof of Case A by considering $Y=\left\{\varphi_{1}(1)\right\}$.

Theorem 3.3 Let $X$ be a simple closed curve and let $p, q \in X$ such that $p \neq q$. Then $\{p, q\}$ makes a hole with respect to multicoherence degree in $\mathcal{F}_{2}(X)$.

Proof. First, we are going to prove that $A=\{(1,0),(-1,0)\} \subset S^{1}$ makes a hole with respect to multicoherence degree in $\mathcal{F}_{2}\left(S^{1}\right)$. By Theorem $2.8, r\left(\mathcal{F}_{2}\left(S^{1}\right)\right)=1$. So, it suffices to show that there exist two closed connected subsets $\mathcal{K}$ and $\mathcal{L}$ of $\mathcal{F}_{2}\left(S^{1}\right)-\{A\}$ such that $\mathcal{F}_{2}\left(S^{1}\right)-\{A\}=\mathcal{K} \cup \mathcal{L}$ and $b_{0}(\mathcal{K} \cap \mathcal{L}) \geq 2$.

Define $\varphi:[0,1] \rightarrow S^{1}$ by $\varphi(t)=(\cos (2 \pi t), \sin (2 \pi t))$ and $\psi: \Delta \rightarrow \mathcal{F}_{2}\left(S^{1}\right)$ by $\psi(t, s)=\{\varphi(t), \varphi(s)\}$. Notice that $\psi$ is well defined and it is surjective. The continuity of $\psi$ follows from that of $\varphi$. Put $\Gamma_{1}=\left\{(u, v) \in \Delta-\left\{\left(0, \frac{1}{2}\right)\right\}: \frac{1}{2}-u \leq\right.$ $v \leq 1-u\}, \Gamma_{2}=\left\{(u, v) \in \Delta-\left\{\left(\frac{1}{2}, 1\right)\right\}: \frac{3}{2}-u \leq v\right\}, \Gamma_{3}=\left\{(u, v) \in \Delta-\left\{\left(0, \frac{1}{2}\right)\right\}: v \leq \frac{1}{2}-u\right\}, \Gamma_{4}=\left\{(u, v) \in \Delta-\left\{\left(\frac{1}{2}, 1\right)\right\}:\right.$ $\left.1-u \leq v \leq \frac{3}{2}-u\right\}, \mathcal{K}=\psi\left(\Gamma_{1}\right) \cup \psi\left(\Gamma_{2}\right)$ and $\mathcal{L}=\psi\left(\Gamma_{3}\right) \cup \psi\left(\Gamma_{4}\right)$.

It is easy to prove that $\mathcal{K}$ and $\mathcal{L}$ are closed subset of $\mathcal{F}_{2}\left(S^{1}\right)-\{A\}$. Clearly, $\mathcal{K} \cup \mathcal{L} \subset \mathcal{F}_{2}\left(S^{1}\right)-\{A\}$. Now, we will prove that $\mathcal{F}_{2}\left(S^{1}\right)-\{A\} \subset \mathcal{K} \cup \mathcal{L}$. Let $\{x, y\} \in \mathcal{F}_{2}\left(S^{1}\right)-\{A\}$ and let $t, s \in[0,1]$ such that $\varphi(t)=x$ and $\varphi(s)=y$. Without loss of generality, we may suppose that $t \leq s$. So, since $\psi(t, s)=\{x, y\}$ and $(t, s) \in \Delta-\left\{\left(0, \frac{1}{2}\right),\left(\frac{1}{2}, 1\right)\right\}=\Gamma_{1} \cup \Gamma_{2} \cup \Gamma_{3} \cup \Gamma_{4}$, $\{x, y\} \in \psi\left(\Gamma_{1}\right) \cup \psi\left(\Gamma_{2}\right) \cup \psi\left(\Gamma_{3}\right) \cup \psi\left(\Gamma_{4}\right)=\mathcal{K} \cup \mathcal{L}$. Thus, $\mathcal{F}_{2}\left(S^{1}\right)-\{A\}=\mathcal{K} \cup \mathcal{L}$.

The connectedness of $\mathcal{K}$ and $\mathcal{L}$ follows from the facts that $\psi(0,0)=\psi(0,1)=\psi(1,1) \in \psi\left(\Gamma_{1}\right) \cap \psi\left(\Gamma_{2}\right) \cap \psi\left(\Gamma_{3}\right) \cap \psi\left(\Gamma_{4}\right)$ and each $\psi\left(\Gamma_{i}\right)$ is connected.

Now, we are going to prove that $b_{0}(\mathcal{K} \cap \mathcal{L}) \geq 2$. Put $\Lambda_{1}=\{(u, v) \in \Delta: v=1-u\}, \Lambda_{2}=\left\{(u, v) \in \Delta-\left\{\left(0, \frac{1}{2}\right)\right\}\right.$ : $\left.v=\frac{1}{2}-u\right\}$ and $\Lambda_{3}=\left\{(u, v) \in \Delta-\left\{\left(\frac{1}{2}, 1\right)\right\}: v=\frac{3}{2}-u\right\}$. Notice that $\Lambda_{1}=\Gamma_{1} \cap \Gamma_{4}, \Lambda_{2}=\Gamma_{1} \cap \Gamma_{3}, \Lambda_{3}=\Gamma_{2} \cap \Gamma_{4}$ and, $\Lambda_{1}, \Lambda_{2}$ and $\Lambda_{3}$ are mutually disjoint. It is easy to see that $\psi\left(\Lambda_{1}\right)=\psi\left(\Gamma_{1} \cap \Gamma_{4}\right)=\psi\left(\Gamma_{1}\right) \cap \psi\left(\Gamma_{4}\right), \psi\left(\Lambda_{2}\right)=$ $\psi\left(\Gamma_{1} \cap \Gamma_{3}\right)=\psi\left(\Gamma_{1}\right) \cap \psi\left(\Gamma_{3}\right), \psi\left(\Lambda_{3}\right)=\psi\left(\Gamma_{2} \cap \Gamma_{4}\right)=\psi\left(\Gamma_{2}\right) \cap \psi\left(\Gamma_{4}\right)$ and $\psi\left(\Gamma_{2}\right) \cap \psi\left(\Gamma_{3}\right)=\emptyset$. We will show that $\psi\left(\Lambda_{1}\right)$, $\psi\left(\Lambda_{2}\right)$ and $\psi\left(\Lambda_{3}\right)$ are the components of $\mathcal{K} \cap \mathcal{L}$. First, notice that $\psi\left(\Lambda_{1}\right) \subset \mathcal{K} \cap \mathcal{L}$ since $\psi\left(\Gamma_{1}\right) \subset \mathcal{K}$ and $\psi\left(\Gamma_{4}\right) \subset \mathcal{L}$. Similarly, it can be proved that $\psi\left(\Lambda_{2}\right)$ and $\psi\left(\Lambda_{3}\right)$ is contained in $\mathcal{K} \cap \mathcal{L}$. Now, to verify that $\mathcal{K} \cap \mathcal{L} \subset \bigcup_{i=1}^{3} \varphi\left(\Lambda_{i}\right)$, let $\{x, y\} \in \mathcal{K} \cap \mathcal{L}$. Since $\mathcal{K}=\psi\left(\Gamma_{1}\right) \cup \psi\left(\Gamma_{2}\right)$, either $\{x, y\} \in \psi\left(\Gamma_{1}\right) \cap \mathcal{L}$ or $\{x, y\} \in \psi\left(\Gamma_{2}\right) \cap \mathcal{L}$. From the facts that $\mathcal{L}=\psi\left(\Gamma_{3}\right) \cup \psi\left(\Lambda_{4}\right)$ and $\psi\left(\Gamma_{2}\right) \cap \psi\left(\Gamma_{3}\right)=\emptyset$, we have $\{x, y\} \in\left(\psi\left(\Gamma_{1}\right) \cap \psi\left(\Gamma_{3}\right)\right) \cup\left(\psi\left(\Gamma_{1}\right) \cap \psi\left(\Gamma_{4}\right)\right) \cup\left(\psi\left(\Gamma_{2}\right) \cap \psi\left(\Gamma_{4}\right)\right)$. So, $\{x, y\} \in \bigcup_{i=1}^{3} \psi\left(\Lambda_{i}\right)$. Finally, since $\Lambda_{1}, \Lambda_{2}$ and $\Lambda_{3}$ are connected and mutually disjoint, $\psi\left(\Lambda_{1}\right), \psi\left(\Lambda_{2}\right)$ and $\psi\left(\Lambda_{3}\right)$ are also connected and mutually disjoint. This proves that $b(\mathcal{K} \cap \mathcal{L})+1=3$.

So, $\mathcal{K}$ and $\mathcal{L}$ satisfy the required properties.

We are ready to prove that $\{p, q\}$ makes a hole with respect to multicoherence degree in $\mathcal{F}_{2}(X)$. Since $X$ is a simple closed curve, there exists a homeomorphism $h: S^{1} \rightarrow X$ such that $h(A)=\{p, q\}$. Consider the induced mapping $h_{2}: \mathcal{F}_{2}\left(S^{1}\right) \rightarrow \mathcal{F}_{2}(X)$ defined by $h_{2}(B)=h(B)$ for each $B \in \mathcal{F}_{2}\left(S^{1}\right)$. By (Higuera \& Illanes, 2011, Theorem 3.1, p. 369), $h_{2}$ is a homeomorphism. Then, since $A$ makes a hole with respect to multicoherence degree in $\mathcal{F}_{2}\left(S^{1}\right)$ and $h_{2}(A)=\{p, q\},\{p, q\}$ makes a hole with respect to multicoherence degree in $\mathcal{F}_{2}(X)$.

Theorem 3.4 Let $X$ be a theta curve and let $p, q \in X$ such that $\operatorname{ord}(p, X)=\operatorname{ord}(q, X)=2$ and $X-\{p, q\}$ is connected. Then $\{p, q\}$ makes a hole with respect to multicoherence degree in $\mathcal{F}_{2}(X)$. 
Proof. Clearly, $X$ is a cyclicly connected graph. Then, by Theorem 2.8, $r\left(\mathcal{F}_{2}(X)\right)=1$. So, to show that $r\left(\mathcal{F}_{2}(X)-\right.$ $\{\{p, q\}\})>1$, we are going to prove that there exist connected closed subsets $\mathcal{K}$ and $\mathcal{L}$ of $\mathcal{F}_{2}(X)-\{\{p, q\}\}$ satisfying $\mathcal{F}_{2}(X)-\{\{p, q\}\}=\mathcal{K} \cup \mathcal{L}$ and $b_{0}(\mathcal{K} \cap \mathcal{L}) \geq 2$.

Put $\mathcal{I}(X)=\left\{I_{1}, I_{2}, I_{3}\right\}$. Without loss of generality, we may assume that $p \in I_{1}$ and $q \in I_{2}$. Given $k \in\{1,2,3\}$, fix a homeomorphism $\varphi_{k}:[0,1] \rightarrow I_{k}$ such that $\varphi_{1}(0)=\varphi_{2}(0)=\varphi_{3}(0)$. We may assume that $\varphi_{1}\left(\frac{1}{2}\right)=p$ and $\varphi_{2}\left(\frac{1}{2}\right)=q$. Notice that $\varphi_{1}(1)=\varphi_{2}(1)=\varphi_{3}(1)$. Put $w=\varphi_{1}(0)$ and $z=\varphi_{1}(1)$. So, $R(X)=\{w, z\}$. Now, for each $k, j \in\{1,2,3\}$, consider $\pi_{k}: \Delta \rightarrow \mathcal{F}_{2}\left(I_{k}\right)$ and $\psi_{(k, j)}:[0,1]^{2} \rightarrow\left\langle I_{k}, I_{j}\right\rangle$ defined by $\pi_{k}(t, s)=\left\{\varphi_{k}(t), \varphi_{k}(s)\right\}$ for each $(t, s) \in \Delta$ and $\psi_{(k, j)}(u, v)=\left\{\varphi_{k}(u), \varphi_{j}(v)\right\}$ for each $(u, v) \in[0,1]^{2}$. Now, let $\Lambda_{1}=\left\{(u, v) \in\left[0, \frac{1}{2}\right] \times\left[\frac{1}{2}, 1\right]-\{\boldsymbol{a}\}\right.$ : $\left.\frac{1}{2} \leq v \leq \frac{3-2 u}{4}\right\}, \Lambda_{2}=\left(\left(\left[\frac{1}{2}, \frac{3}{4}\right] \times\left[\frac{1}{2}, 1\right]\right) \cup\left(\left[\frac{1}{2}, 1\right] \times\left[\frac{1}{2}, \frac{3}{4}\right]\right)\right)-\{\boldsymbol{a}\}, \Lambda_{3}=\left\{(u, v) \in\left[\frac{1}{2}, 1\right] \times\left[0, \frac{1}{2}\right]-\{\boldsymbol{a}\}: v \leq \frac{4-3 u}{2}\right\}$, $\Omega_{1}=\left\{(u, v) \in\left[0, \frac{1}{2}\right] \times\left[\frac{1}{2}, 1\right]-\{\boldsymbol{a}\}: \frac{3-2 u}{4} \leq v\right\}, \Omega_{2}=\left[\frac{3}{4}, 1\right] \times\left[\frac{3}{4}, 1\right], \Omega_{3}=\left\{(u, v) \in\left[\frac{1}{2}, 1\right] \times\left[0, \frac{1}{2}\right]-\{\boldsymbol{a}\}: \frac{3-4 u}{2} \leq v\right\}$ and $\Omega_{4}=\left[0, \frac{1}{2}\right] \times\left[0, \frac{1}{2}\right]-\{\boldsymbol{a}\}$ where $\boldsymbol{a}=\left(\frac{1}{2}, \frac{1}{2}\right)$. Consider $\Gamma_{1}=\left\{(u, v) \in \Delta: \frac{1}{2}-u \leq v \leq \frac{3}{4}-u\right\}, \Gamma_{2}=\{(u, v) \in \Delta$ : $\left.\frac{3}{2}-u \leq v \leq \frac{7}{4}-u\right\}, \Sigma_{1}=\left\{(u, v) \in \Delta: v \leq \frac{1}{2}-u\right\}, \Sigma_{2}=\left\{(u, v) \in \Delta: \frac{3}{4}-u \leq v \leq \frac{3}{2}-u\right\}$ and $\Sigma_{3}=\{(u, v) \in \Delta$ : $\left.\frac{7}{4}-u \leq v\right\}$. Put $\Lambda=\Lambda_{1} \cup \Lambda_{2} \cup \Lambda_{3}, \Omega=\Omega_{1} \cup \Omega_{2} \cup \Omega_{3} \cup \Omega_{4}, \Gamma=\Gamma_{1} \cup \Gamma_{2}$ and $\Sigma=\Sigma_{1} \cup \Sigma_{2} \cup \Sigma_{3}$

For each $k \in\{1,2\}$, let $\mathcal{K}_{k}=\psi_{(k, 3)}\left(\left[\frac{1}{2}, \frac{3}{4}\right] \times[0,1]\right), \mathcal{L}_{k}^{1}=\psi_{(k, 3)}\left(\left[0, \frac{1}{2}\right] \times[0,1]\right)$ and $\mathcal{L}_{k}^{2}=\psi_{(k, 3)}\left(\left[\frac{3}{4}, 1\right] \times[0,1]\right)$.

Define

$$
\begin{gathered}
\mathcal{K}=\psi_{(1,2)}(\Lambda) \cup \pi_{1}(\Gamma) \cup \pi_{2}(\Gamma) \cup \mathcal{K}_{1} \cup \mathcal{K}_{2} \text { and } \\
\mathcal{L}=\psi_{(1,2)}(\Omega) \cup \pi_{1}(\Sigma) \cup \pi_{2}(\Sigma) \cup \mathcal{L}_{1}^{1} \cup \mathcal{L}_{2}^{1} \cup \mathcal{L}_{1}^{2} \cup \mathcal{L}_{2}^{2} \cup \mathcal{F}_{2}\left(I_{3}\right) .
\end{gathered}
$$

It is easy to see that $\mathcal{K}$ and $\mathcal{L}$ are closed subset of $\mathcal{F}_{2}(X)-\{\{p, q\}\}$. In order to prove that $\mathcal{F}_{2}(X)-\{\{p, q\}\} \subset \mathcal{K} \cup \mathcal{L}$, let $\{x, y\} \in \mathcal{F}_{2}(X)-\{\{p, q\}\}$. First, since $X=I_{1} \cup I_{2} \cup I_{3}, \mathcal{F}_{2}(X)=\mathcal{F}_{2}\left(I_{1}\right) \cup \mathcal{F}_{2}\left(I_{2}\right) \cup \mathcal{F}_{2}\left(I_{3}\right) \cup\left\langle I_{1}, I_{2}\right\rangle \cup\left\langle I_{1}, I_{3}\right\rangle \cup\left\langle I_{2}, I_{3}\right\rangle$. Now, notice that $\mathcal{F}_{2}\left(I_{1}\right)=\pi_{1}(\Gamma \cup \Sigma), \mathcal{F}_{2}\left(I_{2}\right)=\pi_{2}(\Gamma \cup \Sigma), \mathcal{F}_{2}\left(I_{3}\right) \subset \mathcal{L},\left\langle I_{1}, I_{2}\right\rangle-\{\{p, q\}\}=\psi_{(1,2)}(\Gamma \cup \Omega),\left\langle I_{1}, I_{3}\right\rangle=\mathcal{K}_{1} \cup \mathcal{L}_{1}^{1} \cup \mathcal{L}_{1}^{2}$ and $\left\langle I_{2}, I_{3}\right\rangle=\mathcal{K}_{2} \cup \mathcal{L}_{2}^{1} \cup \mathcal{L}_{2}^{2}$. Hence, $\{x, y\} \in \mathcal{K} \cup \mathcal{L}$. This proves that $\mathcal{F}_{2}(X)-\{\{p, q\}\}=\mathcal{K} \cup \mathcal{L}$.

To prove that $\mathcal{K}$ and $\mathcal{L}$ are connected, put $\mathfrak{C}=\left\{\Lambda_{1}, \Lambda_{2}, \Lambda_{3}, \Omega_{1}, \Omega_{2}, \Omega_{3}, \Omega_{4}\right\}, \mathfrak{D}=\left\{\Gamma_{1}, \Gamma_{2}, \Sigma_{1}, \Sigma_{2}, \Sigma_{3}\right\}, \mathfrak{F}=\left\{\left[\frac{1}{2}, \frac{3}{4}\right] \times\right.$ $\left.[0,1],\left[0, \frac{1}{2}\right] \times[0,1],\left[\frac{3}{4}, 1\right] \times[0,1]\right\}$ and $\mathfrak{G}=\mathfrak{C} \cup \mathfrak{D} \cup \mathfrak{F}$. It is easy to see that each element of $\mathfrak{G}$ is connected. So, $\psi_{(1,2)}(\Theta), \pi_{k}(\Psi)$ and $\psi_{(k, 3)}(\Upsilon)$ are connected for each $(\Theta, \Psi, \Upsilon, k) \in \mathfrak{C} \times \mathfrak{D} \times \mathfrak{F} \times\{1,2\}$. Notice that $\{w, p\} \in \pi_{1}\left(\Gamma_{1}\right) \cap$ $\psi_{(1,2)}\left(\Lambda_{3}\right) \cap \mathcal{K}_{1},\{p, z\} \in \pi_{1}\left(\Gamma_{2}\right) \cap \psi_{(1,2)}\left(\Lambda_{2}\right) \cap \mathcal{K}_{1},\{q, z\} \in \psi_{(1,2)}\left(\Lambda_{2}\right) \cap \pi_{2}\left(\Gamma_{2}\right) \cap \mathcal{K}_{2}$ and $\{w, q\} \in \psi_{(1,2)}\left(\Lambda_{1}\right) \cap \pi_{2}\left(\Gamma_{1}\right) \cap \mathcal{K}_{2}$. Then, $\mathcal{K}$ is connected. Now, since $\{w\} \in \pi_{1}\left(\Sigma_{1}\right) \cap \pi_{2}\left(\Sigma_{1}\right) \cap \psi_{(1,2)}\left(\Omega_{4}\right) \cap \mathcal{L}_{1}^{1} \cap \mathcal{L}_{2}^{1} \cap \mathcal{F}_{2}\left(I_{3}\right),\{z\} \in \pi_{1}\left(\Sigma_{3}\right) \cap \pi_{2}\left(\Sigma_{3}\right) \cap$ $\psi_{(1,2)}\left(\Omega_{2}\right) \cap \mathcal{L}_{1}^{2} \cap \mathcal{L}_{2}^{2} \cap \mathcal{F}_{2}\left(I_{3}\right)$ and $\{w, z\} \in \pi_{1}\left(\Sigma_{2}\right) \cap \pi_{2}\left(\Sigma_{2}\right) \cap \psi_{(1,2)}\left(\Omega_{1}\right) \cap \psi_{(1,2)}\left(\Omega_{3}\right) \cap \mathcal{L}_{1}^{1} \cap \mathcal{L}_{1}^{2} \cap \mathcal{L}_{2}^{1} \cap \mathcal{L}_{2}^{2} \cap \mathcal{F}_{2}\left(I_{3}\right)$, $\mathcal{L}$ is connected.

Finally, we are going to show that $b_{0}(\mathcal{K} \cap \mathcal{L}) \geq 2$. Given $(n, m) \in\{1,2\} \times\{1,2,3\}$, let $\Pi_{(n, m)}=\Gamma_{n} \cap \Sigma_{m}$. Define $\Upsilon_{(i, j)}=$ $\Lambda_{i} \cap \Omega_{j}$ for each $(i, j) \in\{1,2,3\} \times\{1,2,3,4\}$. For each $k \in\{1,2\}$, consider $\mathcal{H}_{k}=\mathcal{L}_{k}^{1} \cap \mathcal{K}_{k}$ and $\mathcal{J}_{k}=\mathcal{L}_{k}^{2} \cap \mathcal{K}_{k}$. Let $\mathcal{C}_{1}=$ $\pi_{1}\left(\Pi_{(1,1)}\right) \cup \psi_{(1,2)}\left(\Upsilon_{(3,4)}\right) \cup \mathcal{H}_{1} \cup \pi_{1}\left(\Pi_{(2,2)}\right) \cup \psi_{(1,2)}\left(\Upsilon_{(2,1)}\right), C_{2}=\pi_{2}\left(\Pi_{(1,1)}\right) \cup \psi_{(1,2)}\left(\Upsilon_{(1,4)}\right) \cup \mathcal{H}_{2} \cup \pi_{2}\left(\Pi_{(2,2)}\right) \cup \psi_{(1,2)}\left(\Upsilon_{(2,3)}\right)$ and $C_{3}=\pi_{1}\left(\Pi_{(1,2)}\right) \cup \psi_{(1,2)}\left(\Upsilon_{(3,3)}\right) \cup \mathcal{J}_{1} \cup \pi_{1}\left(\Pi_{(2,3)}\right) \cup \psi_{(1,2)}\left(\Upsilon_{(2,2)}\right) \cup \pi_{2}\left(\Pi_{(2,3)}\right) \cup \mathcal{J}_{2} \cup \pi_{2}\left(\Pi_{(1,2)}\right) \cup \psi_{(1,2)}\left(\Upsilon_{(1,1)}\right)$. We are going to prove that $C_{1}, C_{2}$ and $C_{3}$ are the components of $\mathcal{K} \cap \mathcal{L}$.

The following properties are easy to verify,

i) $\Pi_{(n, m)}$ is connected and $\pi_{k}\left(\Pi_{(n, m)}\right)=\pi_{k}\left(\Gamma_{n}\right) \cap \pi_{k}\left(\Sigma_{m}\right)$ for each $n, k \in\{1,2\}$ and $m \in\{n, n+1\}$,

ii) if $\Upsilon_{(i, j)} \neq \emptyset$, then $\Upsilon_{(i, j)}$ is connected and $\psi_{(1,2)}\left(\Upsilon_{(i, j)}\right)=\psi_{(1,2)}\left(\Lambda_{i}\right) \cap \psi_{(1,2)}\left(\Omega_{j}\right)$,

iii) $\mathcal{H}_{k}$ and $J_{k}$ are connected for each $k \in\{1,2\}$,

iv) $\{w, p\} \in \pi_{1}\left(\Pi_{(1,1)}\right) \cap \psi_{(1,2)}\left(\Upsilon_{(3,4)}\right) \cap \mathcal{H}_{1}$,

v) $\{p, z\} \in \mathcal{H}_{1} \cap \pi_{1}\left(\Pi_{(2,2)}\right) \cap \psi_{(1,2)}\left(\Upsilon_{(2,1)}\right)$,

vi) $\{w, q\} \in \pi_{2}\left(\Pi_{(1,1)}\right) \cap \psi_{(1,2)}\left(\Upsilon_{(1,4)}\right) \cap \mathcal{H}_{2}$,

vii) $\{q, z\} \in \mathcal{H}_{2} \cap \pi_{2}\left(\Pi_{(2,2)}\right) \cap \psi_{(1,2)}\left(\Upsilon_{(2,3)}\right)$,

viii) $\left\{w, \varphi_{1}\left(\frac{3}{4}\right)\right\} \in \pi_{1}\left(\Pi_{(1,2)}\right) \cap \psi_{(1,2)}\left(\Upsilon_{(3,3)}\right) \cap \mathcal{J}_{1}$,

ix) $\left\{\varphi_{1}\left(\frac{3}{4}\right), z\right\} \in \mathcal{J}_{1} \cap \pi_{1}\left(\Pi_{(2,3)}\right) \cap \psi_{(1,2)}\left(\Upsilon_{(2,2)}\right)$,

x) $\left\{\varphi_{2}\left(\frac{3}{4}\right), z\right\} \in \psi_{(1,2)}\left(\Upsilon_{(2,2)}\right) \cap \pi_{2}\left(\Pi_{(2,3)}\right) \cap \mathcal{J}_{2}$,

xi) $\left\{w, \varphi_{2}\left(\frac{3}{4}\right)\right\} \in \mathcal{J}_{2} \cap \pi_{2}\left(\Pi_{(1,2)}\right) \cap \psi_{(1,2)}\left(\Upsilon_{(1,1)}\right)$,

xii) $\prod_{\left(n_{1}, m_{1}\right)} \cap \prod_{\left(n_{2}, m_{2}\right)}=\emptyset$ for each $\left(n_{1}, m_{1}\right) \neq\left(n_{2}, m_{2}\right) \in\{1,2\} \times\{1,2,3\}$, 
xiii) $\Upsilon_{\left(i_{1}, j_{1}\right)} \cap \Upsilon_{\left(i_{2}, j_{2}\right)}=\emptyset$ for each $\left(i_{1}, j_{1}\right) \neq\left(i_{2}, j_{2}\right) \in\{1,2,3\} \times\{1,2,3,4\}$,

xiv) $\mathcal{H}_{k} \cap \mathcal{J}_{k}=\emptyset$ for each $k \in\{1,2\}$, and

$\mathrm{xv}) \mathcal{K} \cap \mathcal{L}=\bigcup\left\{\pi_{k}\left(\Pi_{(n, m)}\right):(k, n, m) \in\{1,2\} \times\{1,2\} \times\{1,2,3\}\right\} \cup \bigcup\left\{\psi_{(1,2)}\left(\Upsilon_{(i, j)}\right):(i, j) \in\{1,2,3\} \times\{1,2,3,4\}\right\} \cup$ $\bigcup\left\{\mathcal{H}_{k} \cup \mathcal{J}_{k}: k \in\{1,2\}\right\}$.

The connectedness of $C_{1}, C_{2}$ and $C_{3}$ follows from i)-xi). Using xii)-xiv), it can be proved that $C_{1}, C_{2}$ and $C_{3}$ are mutually disjoint. Finally, from xv), it follows that $\mathcal{K} \cap \mathcal{L}=C_{1} \cup C_{2} \cup C_{3}$.

So, $\mathcal{K}$ and $\mathcal{L}$ satisfy the required properties.

Theorem 3.5 Let $X$ be a cyclicly connected graph and $p, q \in X$. If $p \neq q$, then $\{p, q\}$ makes a hole with respect to multicoherence degree in $\mathcal{F}_{2}(X)$.

Proof. In the case that $X$ is a simple closed curve, the result follows from Theorem 3.3. Now, suppose that $X$ is not a simple closed curve. Since $X$ is a graph, by (Borsuk \& Ulam, 1931, (a), p. 877), $\mathcal{F}_{2}(X)$ is a locally connected space. Then, $\mathcal{F}_{2}(X)-\{\{p, q\}\}$ is a locally connected metric space. So, by (Eilenberg, 1936, Theorem 4, p. 162) and (Stone, 1950, Theorem 5, p. 472), it suffices to show that there exists a retract $\mathcal{Z}$ of $\mathcal{F}_{2}(X)-\{\{p, q\}\}$ such that $r(\mathcal{Z})>r\left(\mathcal{F}_{2}(X)\right)=1$ (see Theorem 2.8). We consider two cases.

Case I. $X-\{p, q\}$ is not connected.

By Lemma 2.3, there exists a simple closed curve $S$ in $X$ containing $p$ and $q$ and a retraction $f: X \rightarrow S$ such that $f^{-1}(p)=\{p\}$ and $f^{-1}(q)=\{q\}$. Put $\mathcal{Z}=\mathcal{F}_{2}(S)-\{\{p, q\}\}$. Since $S$ is a cyclicly connected graph, $r\left(\mathcal{F}_{2}(S)\right)=1$ (see Theorem 2.8). So, by Theorem 3.3,r(Z) $\geq 2$. Finally, define $\bar{f}: \mathcal{F}_{2}(X)-\{\{p, q\}\} \rightarrow \mathcal{Z}$ as follows: for each $A \in \mathcal{F}_{2}(X)-\{\{p, q\}\}$, let $\bar{f}(A)=f(A)$. Using the fact that $f^{-1}(p)=\{p\}$ and $f^{-1}(q)=\{q\}$, it can be proved that $\bar{f}$ is well defined. Since $f$ is continuous, $\bar{f}$ is continuous. Finally, notice that $\bar{f}(B)=B$ for each $B \in \mathcal{Z}$. Thus, $\bar{f}$ is a retraction.

Case II. $X-\{p, q\}$ is connected.

There exists a theta curve $Y$ in $X$ such that $p, q \in Y$ and a retraction $f: X \rightarrow Y$ satisfying $f^{-1}(p)=\{p\}$ and $f^{-1}(q)=\{q\}$ (see Lemma 2.4). Since $X-\{p, q\}$ is connected, $Y-\{p, q\}$ is also connected. Put $\mathcal{Z}=\mathcal{F}_{2}(Y)-\{\{p, q\}$. By Theorem 3.4, $r(\mathcal{Z}) \geq 2$ since $r\left(\mathcal{F}_{2}(Y)\right)=1$ (see Theorem 2.8). Now, define $\bar{f}: \mathcal{F}_{2}(X)-\{\{p, q\}\} \rightarrow \mathcal{Z}$ by $\bar{f}(A)=f(A)$ for each $A \in \mathcal{F}_{2}(X)-\{\{p, q\}\}$. Notice that $\bar{f}$ is well defined since $f^{-1}(p)=\{p\}$ and $f^{-1}(q)=\{q\}$. The continuity of $\bar{f}$ follows from the fact that $f$ is continuous. It is easy to verify that $\bar{f}(B)=B$ for each $B \in \mathcal{Z}$. Thus, $\mathcal{Z}$ is a retract of $\mathcal{F}_{2}(X)-\{\{p, q\}\}$.

\subsection{Classification}

Theorem 3.6 Let $X$ be a cyclicly connected graph and let $p, q \in X$. Then, $\{p, q\}$ makes a hole with respect to multicoherence degree in $\mathcal{F}_{2}(X)$ if and only if either $p=q$ and $p \in R(X)$, or $p \neq q$.

Proof. From (Nadler, Jr., 1992, Theorem 9.10, p. 144; Kuratowski, 1968, Theorem 3, p. 278) and (Nadler, Jr., 1992, Corollary 9.6, p. 142), it follows that $E(X)=\emptyset$. Then, $p, q \in O(X) \cup R(X)$

Assume that $\{p, q\}$ makes a hole in $\mathcal{F}_{2}(X)$. Now, by Theorem 3.1, either $p=q$ and $p \notin O(X)$, or $p \neq q$. So, eihter $p=q$ and $p \in R(X)$ or $p \neq q$. This proves the necessity.

Finally, the sufficiency follows from Theorems 3.2 and 3.5 .

\section{References}

Anaya, J. G. (2007). Making holes in hyperspaces. Top. Appl., 154, 2000-2008. http://dx.doi.org/10.1016/j.topol.2006.09.017

Anaya, J. G. (2011). Making holes in the hyperspace of subcontinua of a Peano continuum. Top. Proc., 37, 1-15.

Anaya, J. G., Maya, D., \& Orozco-Zitli, F. (2010). Agujeros en el segundo producto simétrico de subcontinuos del continuo figura 8. Ciencia Ergosum, 17(3), 307-312.

Anaya, J. G., Maya, D., \& Orozco-Zitli, F. (2012). Making holes in the second symmetric product of dendrites and some fans. Ciencia Ergosum, 19(1), 83-92.

Borsuk, K., \& Ulam, S. (1931). On symmetric products of topological spaces. Bull. Amer. Math. Soc., 37, 875-882. http://dx.doi.org/10.1090/S0002-9904-1931-05290-3 
Eilenberg, S. (1936). Sur les espaces multicohérents I. Fund. Math., 27(1), 153-190.

Higuera, G., \& Illanes, A. (2011). Induced mapping on symmetric product. Top. Proc., 37, 367-401.

Illanes, A. (1985). Multicoherence in symmetric products. An. Inst. Mat. Univ. Nac. Autónoma México, 25, 11-24.

Kuratowski, K. (1968). Topology (Vol. II). New York, London and Warszawa: Academic Press and PWN.

Martínez-Montejano, J. M. (2002). Non-confluence of natural map of products onto symmetric products. In Continuum Theory (Denton, TX. 1999) (Vol. 230, 229-236). Lecture Notes in Pure and Appl. Math., Dekker, New York. http://dx.doi.org/10.1201/9780203910245.ch16

Michael, E. (1951). Topologies on space of subsets. Trans. Amer. Math. Soc., 71, $152-182$. http://dx.doi.org/10.1090/S0002-9947-1951-0042109-4

Nadler, Jr., S. B. (1992). Continuum Theory: An introduction. Monographs and Textbooks in Pure and Applied Mathematics, 158. New York: Marcel Dekker, Inc.

Stone, A. H. (1950). Incidence relations in multicoherence spaces II. Canadian J. Math., 2, 461-480. http://dx.doi.org/10.4153/CJM-1950-044-5

Whyburn, G. T. (1942). Analytic Topology (Vol. 28). Amer. Math. Soc. Colloq. Publ. (reprinted with corrections 1971).

\section{Copyrights}

Copyright for this article is retained by the author(s), with first publication rights granted to the journal.

This is an open-access article distributed under the terms and conditions of the Creative Commons Attribution license (http://creativecommons.org/licenses/by/3.0/). 